\title{
3-\$27-4 Symposium
}

\section{Analyses of Foxp $3^{+}$Treg cells and Tr1 cells in subcutaneous immunotherapy (SCIT)-treated allergic individuals in humans and mice}

\author{
Masaya Matsuda ${ }^{1}$, Tetsuya Terada $^{2}$, Kazuyuki Kitatani $^{1}$, Ryo Kawata $^{2}$, Takeshi Nabe ${ }^{1}$
}

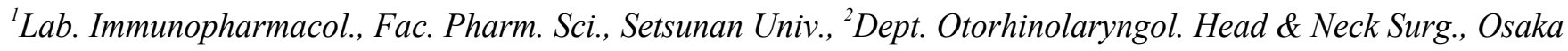
Med. Col.

Mechanisms of allergen immunotherapy have not been fully elucidated. We have analyzed whether numbers of Foxp3 + Treg cells and Tr1 cells (IL-10-producing Foxp3 CD4 ${ }^{+} \mathrm{T}$ cells) are increased in SCIT-treated Japanese cedar pollinosis patients and allergic mice. Peripheral blood mononuclear cells (PBMCs) were collected from the patients treated with or without SCIT. Ovalbumin (OVA)-sensitized mice received s.c. dosages of OVA for SCIT, followed by intratracheal challenges with OVA. The lungs were collected from the allergic mice treated with or without SCIT. The human PBMCs and murine lung cells were stimulated, and analyzed by flow cytometer. In both strains, numbers of Tr1 cells but not Foxp3 ${ }^{+}$Treg cells in SCIT-treated individuals were significantly larger than those in the non-SCITtreated. In mice, SCIT treatment ameliorated allergic airway inflammation such as eosinophilia, hyperresponsiveness and histological changes. In another experiment of mice, Tr1 cells were induced in vitro by culture of splenocytes of sensitized mice with OVA and cytokines, and adoptively transferred to sensitized mice, resulting in effective suppression of the allergic airway inflammation. In conclusion, $\operatorname{Tr} 1$ cells could play roles in clinical effectiveness of SCIT. 